Pacific Journal of Mathematics

THE WHITAKER MODELS OF INDUCED 


\section{THE WHITTAKER MODELS OF INDUCED REPRESENTATIONS}

\section{HERVE JACQUET AND JoSEPH SHALIKA}

If $F$ is a local non-Archimedean field, then every irreducible admissible representation $\pi$ of $\mathrm{GL}(r, F)$ is a quotient of a representation $\xi$ induced by tempered ones. We show that $\xi$ has a Whittaker model, even though it may fail to be irreducible.

\section{Introduction and notations.}

(1.1) Let $F$ be a local non-Archimedean field and $\psi$ an additive character of $F$. Let $G$ be the group $\mathrm{GL}(2, F)$ and $B$ the subgroup of triangular matrices in $G$. If $\mu_{1}$ and $\mu_{2}$ are two characters of $F^{\times}$we may consider the induced representation $\xi=\operatorname{Ind}\left(G, B ; \mu_{1}, \mu_{2}\right)$. There is a nonzero linear form $\lambda$ on the space $V$ of $\xi$ such that

$$
\lambda\left[\xi\left(\begin{array}{cc}
1 & x \\
0 & 1
\end{array}\right) f\right]=\psi(x) \lambda(f), \quad f \in V .
$$

The map which sends $f$ to the function $W$, defined by

$$
W(g)=\lambda[\xi(g) f],
$$

is clearly bijective if $\xi$ is irreducible, that is, if $\mu_{1} \cdot \mu_{2}^{-1} \neq \alpha_{F}^{ \pm 1}$ (we denote by $\alpha_{F}$ or $\alpha$ the module of $F$ ). If $\mu_{1} \cdot \mu_{2}^{-1}=\alpha^{-1}$, the kernel of the map is one dimensional. If $\mu_{1} \cdot \mu_{2}^{-1}=\alpha$ the map has trivial kernel. We recall the proof. Suppose more generally that $\mu_{1} \cdot \mu_{2}^{-1}=\chi \alpha^{u}$ with $\chi \bar{\chi}=1$ and $0<u$. Then we may choose $\lambda$ in such a way that

$$
\left.W\left(\begin{array}{ll}
a & 0 \\
0 & 1
\end{array}\right)=\hat{H(}-a\right) \mu_{2}(a)|a|^{1 / 2}, \quad \hat{H(a)}=\int H(x) \psi(x a) d x,
$$

where $H$ is the element of $L^{1}(F)$ defined by

$$
H(x)=f\left[\left(\begin{array}{ll}
0 & 1 \\
1 & 0
\end{array}\right)\left(\begin{array}{cc}
1 & x \\
0 & 1
\end{array}\right)\right]
$$

From the Fourier inversion formula, $W \mid B$ implies $H=0$ and then, by continuity, $f=0$. Thus we have proved the injectivity of the map $f \mapsto W$ and even the fact that the $W$ 's are determined by their restriction to $B$.

(1.2) In this paper we extend this result (and its proof) to the group $G_{r}=\mathrm{GL}(r, F), r \geq 2$. In a precise way, let $Q$ be the upper standard 
parabolic subgroup of type $\left(r_{1}, r_{2}, \ldots, r_{m}\right), \Sigma r_{i}=r$, in $G_{r}$. Then $Q=M U$ where $U$ is the unipotent radical of $Q$ and $M$ isomorphic to $\Pi \operatorname{GL}\left(r_{i}\right)$. Let $\pi_{i}, 1 \leq i \leq m$, be an irreducible representation of $\mathrm{GL}\left(r_{i}, F\right)$; suppose $\pi_{l}=\pi_{t, 0} \otimes \alpha^{u_{i}}$, where $\pi_{i, 0}$ is irreducible, unitary, tempered and $u_{1}>u_{2}>$ $\cdots>u_{m}$. We refer to the induced representation

$$
\xi=\operatorname{Ind}\left(G_{r}, Q ; \pi_{1}, \pi_{2}, \ldots, \pi_{m}\right)
$$

as an induced representation of "Langlands' type". Let now $N_{r}$ be the group of upper triangular matrices with unit diagonal and let $\theta$ or $\theta_{r}$ be the character of $N_{r}$ defined by

$$
\theta(n)=\prod_{i=1}^{r-1} \psi\left(n_{l, l+1}\right) .
$$

Then there is a nonzero linear form $\lambda$ on the space of $\xi$ and, up to a scalar factor, only one such that

$$
\lambda[\xi(n) f]=\theta(n) \lambda(f) .
$$

Let $\mho(\xi ; \psi)$ be the space spanned by the functions of the form (1.1.1). Our goal is to prove that the map $f \mapsto W$ is bijective, even though $\xi$ may be reducible. In fact we prove a little more: in the terminology of [B-Z] (Theorem 4.9) the representation $\xi$ has a Kirillov model. We remark that when all $\pi_{l, 0}$ are supercuspidal, our result is a special case of Theorem 4.11 in [B-Z]. In general, one can try to reduce our result to theirs by imbedding each $\pi_{i, 0}$ in a representation induced by supercuspidal ones (cf. [Z]). For instance, denote by $B_{r}$ the group of upper-triangular matrices in $G_{r}$ and by $\sigma_{r}$ the (unique) invariant irreducible subspace of

$$
\operatorname{Ind}\left(G_{r}, B_{r} ; \alpha^{(r-1) / 2}, \alpha^{(r-1) / 2-1}, \ldots, \alpha^{-(r-1) / 2}\right) .
$$

Then $\sigma_{r}$ is a square-integrable representation (ordinary special representation). Consider now the induced representation

$$
\xi=\operatorname{Ind}\left(G_{5}, Q ; \sigma_{3} \otimes \alpha^{1 / 2}, \sigma_{2}\right),
$$

where $Q$ has type $(3,2)$. Then $\xi$ is a subrepresentation of

$$
\eta=\operatorname{Ind}\left(G_{5}, B_{5} ; \rho_{1}, \rho_{2}, \ldots, \rho_{5}\right)
$$

where $\rho_{3}=\alpha^{-1 / 2}, \rho_{4}=\alpha^{1 / 2}$. Since $\rho_{4}=\rho_{3} \otimes \alpha$, Theorem 4.11 of [B-Z] does not apply to $\eta$. Thus our result does not follow directly from Theorem 4.11 of [B-Z]; some extra work is needed.

At any rate, our approach is more direct and we use the results of Bernstein-Zelevinski only in an auxiliary way. In more detail, let $P_{r}$ be the 
subgroup of matrices $p$ in $G_{r}$ of the form

$$
p=\left(\begin{array}{ll}
g & * \\
0 & 1
\end{array}\right), \quad g \in G_{r-1} .
$$

Call $\tau_{r}$ the unitary representation of $P_{r}$ induced (in Mackey's sense) by $\theta_{r}$. Then $\tau_{r}$ is irreducible and the right regular representation of $P_{r}$ is a multiple of $\tau_{r}$; the right regular representation of $G_{r}$ has the same property, when restricted to $P_{r}$. Thus, if $\pi$ is an irreducible (preunitary) square-integrable representation, then denoting by $\bar{\pi}$ the corresponding unitary representation, we see that $\bar{\pi} \mid P_{r}$ is a multiple of $\tau_{r}$. (Cf., for instance, $[\mathbf{J}]$ ). Thus $\pi$ is generic, that is, there is a linear form $\lambda \neq 0$ on the space $V$ of $\pi$ satisfying (1.2.3). Since $\lambda$ is unique, within a scalar factor, we see that in fact $\bar{\pi} \mid P_{r} \simeq \tau_{r}$. Finally if $\eta$ is an induced representation of the form

$$
\eta=\operatorname{Ind}\left(G_{r} Q ; \pi_{1}, \pi_{2}, \ldots, \pi_{m}\right),
$$

where the $\pi_{i}$ are irreducible square-integrable, then $\eta$ is pre-unitary and $\bar{\eta} \mid P_{r} \simeq \tau_{r}$ (loc. cit.). In particular $\eta$ is irreducible. This shows that if $\pi$ is any irreducible pre-unitary tempered representation of $G_{r}$ then $\bar{\pi} \mid P_{r} \simeq \tau_{r}$. This is, essentially, all we need to know about tempered representations (cf. $§ 2$ below).

We also remark that the problem of finding all irreducible square-integrable representations of $G_{r}$ is equivalent to the problem of finding all irreducible generic ones. Indeed, if $\pi$ is a square-integrable representation, then $\pi$ is generic by the above remarks, thus by Theorem 9.7 of $[\mathbf{B}-\mathbf{Z}]$ (classification of all generic representations) $\pi$ is equivalent to an induced representation of the form

$$
\xi=\operatorname{Ind}\left(G_{r}, Q ; \sigma_{1}, \sigma_{2}, \ldots, \sigma_{m}\right)
$$

where the $\sigma_{i}$ are "generalized special representations". But then Casselman's criterion for square-integrability shows that, in fact, $\xi$ is itself a generalized special representation: this is a sketch of the proof of Theorem 9.3 stated in $[\mathbf{Z}]$ and due to I. N. Bernstein. Conversely if $\xi$ is a representation of the form (1.2.1) then $\xi$ has a unique irreducible quotient $J\left(\pi_{1}, \pi_{2}, \ldots, \pi_{m}\right)$ (“Langlands' quotient”: cf. [B-W] XI, §2). If $\xi$ is irreducible then our result implies that $J\left(\pi_{1}, \pi_{2}, \ldots, \pi_{m}\right)$ is degenerate (not generic). Since any irreducible representation $\pi$ of $G_{r}$ has the form $J\left(\pi_{1}, \pi_{2}, \ldots, \pi_{r}\right)$ for appropriate $\pi_{l}$, we see that if $\pi$ is generic then $\pi$ must be equivalent to a representation of the form (1.2.1); that is, we have another proof of Theorem 9.7 of [B-Z]. 
Finally we also remark that our result and its proof apply to the case $F=\mathbf{R}$ or $\mathbf{C}$ as well. Naturally $\lambda$ in (1.1.3) and (1.1.1) is then a linear form defined and continuous on an appropriate space of smooth vectors to which $f$ belongs. One needs to duplicate the estimates of $\$ 2$ and check that in (3.1.2), the linear form $f \mapsto W(e)$ can be taken to be $\lambda$, that is, is continuous. Furthermore in (3.2.15) the right-hand side does not have support in the set (3.2.16) but is "of rapid decrease for $\left|a_{i}\right|$ large". Rather than dealing with these minor changes now we prefer to wait for another occasion. We also remark that, taking again into account Langlands' classification and Theorem $\mathrm{D}$ of $[\mathbf{K}]$, we get, for $\operatorname{GL}(r, F)$, another easy proof of the difficult Theorem 6.2 of [V].

However, on the whole, our motivations are global. In [J-P-S] Theorem (13.6) and [G-J], §4 we used this result for GL(3). Similar applications are expected for higher $r$ 's.

(1.3) In addition to the notations already introduced we will use the following ones: $q$ will be the cardinality of the residual field of $F, \Re$ the ring of integers in $F ; K_{r}$ will be the subgroup $\mathrm{GL}(r, \Re)$. We will denote by $Z_{r}$ the center of $G_{r}$, by $A_{r}$ the subgroup of diagonal matrices in $G_{r}$, by $B_{r}=A_{r} N_{r}$ the group of upper triangular matrices and, finally, by $P_{r}$ the subgroup of matrices of the form

$$
p=\left(\begin{array}{ll}
g & * \\
0 & 1
\end{array}\right), \quad g \in G_{r-1} .
$$

\section{Estimate of tempered Whittaker functions.}

(2.1) Let $\pi$ be an irreducible pre-unitary tempered representation of $G_{r}$. Then there is a linear form $\lambda \neq 0$ on the space $V$ of $\pi$ satisfying (1.2.3) and, within a scalar factor, only one. We denote by $\mho \delta(\pi ; \psi)$ the space spanned by functions of the form (1.1.1) with $f$ in $V$. We recall some known facts on the elements of $\mathscr{U}(\pi ; \psi)$.

(2.2) If $W$ is in $\mathscr{W}(\pi ; \psi)$ then the integral

$$
\Psi(s, W, \bar{W}, \Phi)=\int_{N_{r} \backslash G_{r}} W(g) \bar{W}(g) \Phi[(0,0, \ldots, 0,1) g]|\operatorname{det} g|^{s} d g,
$$

where $\Phi$ is in the space $\delta\left(F^{r}\right)$ of Schwartz-Bruhat functions on $F^{r}$, converges for Res $\gg 0$ and represents a rational fraction in $q^{-s}$ without pole for Res $>0$ ([J-P-S] Prop. (8.4)); in passing we note that this result is independent of the classification of all square-integrable representations.

(2.3) The unitary representation of $G_{r}$ corresponding to $\pi$ has the property that its restriction to the subgroup $P_{r}$ is equivalent to the 
representation $\tau_{r}$ of $P_{r}$ induced (in Mackey's sense) by $\theta_{r}$. It amounts to the same to say that

$$
B\left(W, W^{\prime}\right)=\int_{N_{r-1} \backslash G_{r-1}} W\left[\left(\begin{array}{cc}
h & 0 \\
0 & 1
\end{array}\right)\right] \bar{W}^{\prime}\left[\left(\begin{array}{cc}
h & 0 \\
0 & 1
\end{array}\right)\right] d h
$$

defines a $G_{r}$-invariant form on $\mathscr{W}(\pi ; \psi)(\mathrm{cf} .[\mathbf{J}])$. From this or Theorem (4.9) of $[\mathbf{B}-\mathbf{Z}]$ it follows that any $W$ is determined by its restriction to $P_{r}$.

(2.4) Finally, the space of these restrictions contains the space $\mathcal{K}_{0}(\pi ; \psi)$ of functions $f$ on $G_{r}$, transforming on the left under $\theta_{r}$, right smooth and of compact support mod $N_{r}([\mathbf{G}-\mathbf{K}](5.2))$.

(2.5) We need an estimate for the elements of $\mathcal{Q}(\pi ; \psi)$. The quickest proof uses (2.2). Let $\delta_{r}$ denote the module of the Borel subgroup $B_{r}$ in $G_{r}$. We will extend $\delta_{r}$ to a function on $G_{r}$ which is $K_{r}$-invariant on the right. We remark that

$$
\delta_{r}\left[\left(\begin{array}{ll}
g & 0 \\
0 & 1
\end{array}\right)\right]=\delta_{r-1}(g)|\operatorname{det} g|
$$

if $g$ is in $G_{r-1}$. We also define a function $\Lambda_{r}$ on $G_{r}$ by setting

$$
\Lambda_{r}\left(z n\left(\begin{array}{cc}
g & 0 \\
0 & 1
\end{array}\right) k\right)=|\operatorname{det} g|
$$

for $z \in Z_{r}, n \in N_{r}, k \in K_{r}, g \in G_{r-1}$.

Proposition. Suppose $\pi$ is a tempered representation of $G_{r}$ and $W$ is in $\mathcal{Q}(\pi ; \psi)$. Then, for any $s>0$, there is a constant $c_{s}>0$ such that $|W|^{2} \leq$ $c_{s} \delta_{r} \Lambda_{r}^{-s}$.

Proof. Let $\Phi \geq 0$ be an element of $\delta\left(F^{r}\right)$ which is $K_{r}$ invariant on the right. Then, for $s \gg 0$, setting $\eta_{r}=(0,0, \ldots, 0,1)$, we have:

$$
\begin{aligned}
\Psi(s, & W, \bar{W}, \Phi) \\
= & \int_{K_{r}} d k \int_{A_{r-1}}|W|^{2}\left[\left(\begin{array}{cc}
a & 0 \\
0 & 1
\end{array}\right) k\right] \delta_{r}^{-1}\left[\left(\begin{array}{cc}
a & 0 \\
0 & 1
\end{array}\right)\right]|\operatorname{det} a|^{s} d^{\times} a \\
& \times \int_{F^{\times}} \Phi\left[\eta_{r} b k\right]|b|^{r s} d^{\times} b .
\end{aligned}
$$

The convergence of the integral for Res $\gg 0$ amounts to the convergence of a power series in $x=q^{-s}$,

$$
\Psi(s, W, \bar{W}, \Phi)=\sum_{m \geq m_{0}} a_{m} x^{m},
$$


say for $0<|x|<\varepsilon$ (cf. (4.1) and (4.2) in [J-P-S]). By (2.2), the series in (2) actually converges for $0<|x|<1$. But then since the integrand in (1) is $\geq 0$, the integral for $\Psi$ must actually converge for $s>0$. In particular

$$
\int|W|^{2}\left[\left(\begin{array}{cc}
a & 0 \\
0 & 1
\end{array}\right) k\right] \delta_{r}^{-1}\left[\left(\begin{array}{cc}
a & 0 \\
0 & 1
\end{array}\right)\right]|\operatorname{det} a|^{s} d^{\times} a<+\infty
$$

(for $s>0$ ) for all $k \in K_{r}$. Fix $k$ then and let us denote by $f(a), a \in$ $\left(F^{\times}\right)^{r-1} \simeq A_{r-1}$, the integrand in (3). Clearly there is an open compact subgroup, $U$ say, of $\left(F^{\times}\right)^{r-1}$ such that $f(a \varepsilon)=f(a)$ for all $a$ in $\left(F^{\times}\right)^{r-1}, \varepsilon$ in $U$. We deduce at once that, for all $b \in\left(F^{\times}\right)^{r-1}$,

$$
|f(b)| \leq c \int|f(a)| d^{\times} a
$$

$c$ a positive constant. In other words the integrand in (3) is bounded. This is precisely what we wanted to prove.

\section{Induced representations of Langlands' type.}

(3.1) Consider a representation

$$
\xi=\operatorname{Ind}\left(G_{r}, Q ; \pi_{1}, \pi_{2}, \ldots, \pi_{m}\right)
$$

(notations as in (1.2)). A vector $f$ in the space of $\xi$ may be regarded as a function on $G_{r}$ with values in $\otimes_{i=1}^{m} \mathscr{W}\left(\pi_{i} ; \psi\right)$; it may also be regarded as a scalar function on $G_{r} \times G_{r_{1}} \times \cdots \times G_{r_{m}}$ whose value at $\left(g, h_{1}, h_{2}, \ldots, h_{m}\right)$ we denote by $f\left(g ; h_{1}, h_{2}, \ldots, h_{m}\right)$. The integral

$$
W(g)=\int_{U} f(w u g ; e, e, \ldots, e) \bar{\theta}(u) d u,
$$

where

$$
w=\left(\begin{array}{cccc}
0 & & 1_{r_{1}} \\
& & 1_{r_{2}} & \\
1_{r_{m}} & . & & 0
\end{array}\right),
$$

and $d u$ is a Haar-measure on the unipotent radical $U$ of the parabolic subgroup of type $\left(r_{m}, r_{m-1}, \ldots, r_{2}, r_{1}\right)$, defines an element of $थ(\xi ; \psi)$ provided it converges. We are going to show that it converges for all $f$; in fact, we are going to obtain a majorization of the function

$$
h \mapsto \int f(w u g ; e, e, \ldots, e, h) d u .
$$


It will be sufficient to obtain an upper bound for the integral

$$
\int|f|(w u g ; e, e, \ldots, e, h) d u .
$$

This integral, finite or infinite, is equal to

$$
|\operatorname{det} h|^{-\left(r-r_{m}\right) / 2} \int|f|\left[w u\left(\begin{array}{ll}
h & 0 \\
0 & 1_{r-r_{m}}
\end{array}\right) g ; e, e, \ldots, e\right] d u .
$$

With notation as in (2.5), let $f_{0}$ be the function defined by

$$
f_{0}(g)=\delta_{Q}^{1 / 2}(q) \prod_{j=1}^{m} \delta_{r_{j}}^{1 / 2}\left(g_{\jmath}\right) \Lambda_{r_{j}}\left(g_{\jmath}\right)^{-s_{\jmath}}\left|\operatorname{det} g_{\jmath}\right|^{\mu_{\jmath}},
$$

for $g$ of the form $g=q k, q \in Q, k \in K_{r}$ and $q$ of the form

$$
q=\left(\begin{array}{cccc}
g_{1} & & & * \\
& g_{2} & & \\
& & \ddots & \\
0 & & & g_{m}
\end{array}\right), \quad g_{i} \in G_{r_{i}} .
$$

Here $\left(s_{1}, s_{2}, \ldots, s_{m}\right)$ is an $m$-tuple of positive numbers to be chosen below. Next we apply Proposition (2.5) to the (quasi-) tempered representations $\pi_{l}(1 \leq i \leq m)$ to conclude that given $g_{0} \in G_{r}$, there is a constant $c>0$ such that

$$
|f|\left(g g_{0} ; e, e, \ldots, e\right) \leq c f_{0}(g) .
$$

Thus all we need to do is to obtain an upper bound for the function

$$
|\operatorname{det} h|^{-\left(r-r_{m}\right) / 2} \int f_{0}\left[w u\left(\begin{array}{ll}
h & 0 \\
0 & 1_{r-r_{m}}
\end{array}\right)\right] d u \text {. }
$$

This is actually equal to

$$
\int f_{0}(w u) d u \delta_{r_{m}}^{1 / 2}(h)|\operatorname{det} h|^{u_{m}} \Lambda_{r_{m}}(h)^{-s_{m}} .
$$

We are thus reduced to proving that

$$
\int f_{0}(w u) d u<+\infty .
$$

For that let $V$ denote the unipotent radical of the lower parabolic subgroup of $G_{r}$ of type $\left(r_{1}, \ldots, r_{m}\right)$. Then the integral (11) is the same as the integral

$$
\int_{V} f_{0}(v) d v
$$


Next for $q$ a diagonal matrix of the form (8), we have

$$
\delta_{B}(q)=\delta_{Q}(q) \prod_{1 \leq j \leq m} \delta_{r_{j}}\left(g_{j}\right)
$$

from which we see that for $q=\operatorname{diag}\left(a_{1}, a_{2}, \ldots, a_{r}\right)$

$$
\begin{aligned}
f_{0}(q)= & \delta_{B}^{1 / 2}(a)\left|a_{1} a_{2} \cdots a_{r_{1}-1}\right|^{u_{1}-s_{1}}\left|a_{r_{1}}\right|^{\left(r_{1}-1\right) s_{1}+u_{1}} \\
& \cdot\left|a_{r_{1}+1} \cdots a_{r_{1}+r_{2}-1}\right|^{u_{2}-s_{2}}\left|a_{r_{1}+r_{2}}\right|^{\left(r_{2}-1\right) s_{2}+u_{2}} \cdots .
\end{aligned}
$$

We have seen then that to insure the convergence of (13) it suffices to choose the $s_{t}>0$ so that

$$
u_{1}+\left(r_{1}-1\right) s_{1}>u_{1}-s_{1}>u_{2}+\left(r_{2}-1\right) s_{2}>u_{2}-s_{2}>\cdots .
$$

Each inequality in (15) is either true or can be made true by making the $s_{\text {, }}$ positive and sufficiently small. We have now proved that the integral in (2) is indeed convergent and, moreover, obtained the inequality

$$
\int_{U}|f|(w u g ; e, e, \ldots, e, h) d u \leq c_{v} \delta_{r_{m}}^{1 / 2}(h) \Lambda_{r_{m}}(h)^{-v}|\operatorname{det} h|^{u_{m}},
$$

where $v$ is any sufficiently small positive number and $w$ is given by (3).

(3.2) Proposition. Let $\xi$ be the representation (3.1.1). Then the map $f \mapsto W$ from the space of $\xi$ to $\mathscr{W}(\xi ; \psi)$ defined by (3.1.2) is bijective. Moreover, if $W \in \mathcal{U}(\xi ; \psi)$ then the relation $W \mid P_{r}=0$ implies $W=0$.

Proof. Our assertion is trivial for $m=1$. Thus we may assume $m>1$ and our assertion proved for $m-1$. Consider then the induced representation

$$
\xi^{*}=\operatorname{Ind}\left(G_{r}, Q^{*} ; \xi^{\prime}, \pi_{m}\right)
$$

where

$$
\xi^{\prime}=\operatorname{Ind}\left(G_{r-r_{m}}, Q^{\prime} ; \pi_{1}, \pi_{2}, \ldots, \pi_{m-1}\right),
$$

where $Q^{*}$ has type $\left(r-r_{m}, r_{m}\right)$ and $Q^{\prime}$ has type $\left(r_{1}, r_{2}, \ldots, r_{m-1}\right)$. Furthermore, by the induction hypothesis, we may regard $\xi^{\prime}$ as acting on $\mathscr{W}\left(\xi^{\prime} ; \psi\right)$. Thus we may regard an element $f^{*}$ of $\xi^{*}$ as a function on $G_{r}$ with values in $\mathcal{W}\left(\xi^{\prime} ; \psi\right) \otimes \mho\left(\pi_{m} ; \psi\right)$, or as a scalar function on $G_{r} \times$ $G_{r-r_{m}} \times G_{r_{m}}$. We denote its value at $\left(g, h_{1}, h_{2}\right)$ by $f^{*}\left(g ; h_{1}, h_{2}\right)$. Of course the representations $\xi$ and $\xi^{*}$ are equivalent. If $f$, as in (3.1), is in the space of $\xi$ then the exact relation between $f$ and $f^{*}$ is given by

$$
f^{*}[g ; e, e]=\int_{V^{\prime}} f\left[\left(\begin{array}{cc}
w^{\prime} & 0 \\
0 & 1_{r_{m}}
\end{array}\right)\left(\begin{array}{cc}
v & 0 \\
0 & 1_{r_{m}}
\end{array}\right) g ; e, e, \ldots, e\right] \bar{\theta}_{r-r_{m}}(v) d v,
$$


where

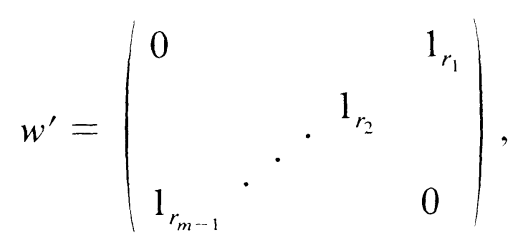

and $V^{\prime}$ is the unipotent radical of the (upper) parabolic in $G_{r-r_{m}}$ of type $\left(r_{m-1}, r_{m-2}, \ldots, r_{1}\right)$. Writing $(11.2)$ as an iterated integral, we readily find that in terms of $f^{*}$,

$$
W(g)=\int_{V^{*}} f^{*}\left[w^{*} v g ; e, e\right] \bar{\theta}_{r}(v) d v
$$

where now

$$
w^{*}=\left(\begin{array}{ll}
0 & 1_{r-r_{m}} \\
1_{r_{m}} & 0
\end{array}\right),
$$

and $V^{*}$ is the unipotent radical of the parabolic in $G_{r}$ of type $\left(r_{m}, r-r_{m}\right)$. Of course the convergence of the integral (3.1.2) implies that of both integrals (3) and (5) (for all $g \in G_{r}$ ). Since the map $f \mapsto f^{*}$ is bijective, all of our assertions will be proved if we show

$$
W \mid P_{r}=0 \text { implies that } f^{*}=0 .
$$

Assume then that $W \mid P_{r}=0$. Explicitly this reads

$$
\int f^{*}\left[w^{*}\left(\begin{array}{ll}
1_{r_{m}} & x \\
0 & 1_{r-r_{m}}
\end{array}\right) p ; e, e\right] \psi(\operatorname{tr}(\varepsilon x)) d x=0
$$

for all $p \in P_{r}$. Here

(9)

$$
\varepsilon=\left(\begin{array}{cccc}
0 & 0 & \cdots & 1 \\
0 & 0 & \cdots & 0 \\
\vdots & \vdots & & \vdots \\
0 & 0 & \cdots & 0
\end{array}\right) \quad\left(r-r_{m} \text { rows, } r_{m} \text { columns }\right)
$$

Replacing $p$ by

$$
\left(\begin{array}{ll}
g_{1} & 0 \\
0 & 1_{r-r_{m}}
\end{array}\right) p
$$


where $g_{1} \in G_{r_{m}}$, and changing variables, we can write this condition in the form

$$
\int f^{*}\left[w^{*}\left(\begin{array}{ll}
1_{r_{m}} & x \\
0 & 1_{r-r_{m}}
\end{array}\right) p ; e, g_{1}\right] \psi\left(\operatorname{tr}\left(\varepsilon g_{1} x\right)\right) d x=0,
$$

for all $p \in P_{r}, g_{1} \in G_{r_{m}}$. We can also replace $g_{1}$ by $h g_{1}$ where $h \in P_{r_{m}}$. Note that $\varepsilon h=\varepsilon$. Thus if we set, for $h \in G_{r_{m}}$,

$$
F(h)=\int f^{*}\left[w^{*}\left(\begin{array}{ll}
1_{r_{m}} & x \\
0 & 1_{r-r_{m}}
\end{array}\right) p ; e, h g_{1}\right] \psi\left(\operatorname{tr}\left(\varepsilon g_{1} x\right)\right) d x,
$$

then we see that the function $F$ defined on $G_{r_{m}}$ has a zero restriction to $P_{r_{m}}$. At this point we may assume $u_{m}=0$. We are going to show that $F$ is actually zero. To see that we first need a majorization of $F$. Using (3) to express $f^{*}$ in terms of $f$ we obtain at once from (3.1.16):

$$
|F(h)| \leq c_{v} \delta_{r_{m}}^{1 / 2}(h) \Lambda_{r_{m}}(h)^{-v},
$$

again for $v>0$ sufficiently small, and all $h \in G_{r_{m}}$.

Thus, for $W^{\prime} \in \mathscr{W}\left(\pi_{m} ; \psi\right)$, we have the inequality

$$
\begin{aligned}
& \int_{N_{r_{m-1}} \backslash G_{r_{m-1}}}\left|F W^{\prime}\right|\left[\left(\begin{array}{cc}
h & 0 \\
0 & 1
\end{array}\right)\right] d h \\
& \leq c_{v} \int_{N_{r_{m-1}} \backslash G_{r_{m-1}}}\left|W^{\prime}\right|\left[\left(\begin{array}{ll}
h & 0 \\
0 & 1
\end{array}\right)\right] \delta_{r_{m}}^{1 / 2}\left[\left(\begin{array}{cc}
h & 0 \\
0 & 1
\end{array}\right)\right] \Lambda_{r_{m}}\left[\left(\begin{array}{ll}
h & 0 \\
0 & 1
\end{array}\right)\right]^{-v} d h .
\end{aligned}
$$

We claim now that both integrals are finite. It suffices to check that the integral

$$
\int_{A_{r_{m-1}}}\left|W^{\prime}\right|\left[\left(\begin{array}{cc}
a & 0 \\
0 & 1
\end{array}\right)\right] \delta_{r_{m-1}}^{-1}(a) \delta_{r_{m}}^{1 / 2}\left[\left(\begin{array}{cc}
a & 0 \\
0 & 1
\end{array}\right)\right]|\operatorname{det} a|^{-v} d^{\times} a
$$

is finite for any $v>0$. Now by (2.5) we have

$$
\left|W^{\prime}\left[\left(\begin{array}{ll}
a & 0 \\
0 & 1
\end{array}\right)\right]\right| \leq c_{v}^{\prime} \delta_{r_{m}}^{1 / 2}\left[\left(\begin{array}{cc}
a & 0 \\
0 & 1
\end{array}\right)\right]|\operatorname{det} a|^{-v} .
$$

Moreover the support of $W^{\prime}\left[\left(\begin{array}{ll}a & 0 \\ 0 & 1\end{array}\right)\right]$ is contained in the set $C$ defined by the conditions

$$
a=\operatorname{diag}\left(a_{1} a_{2} \cdots a_{r-1}, a_{2} \cdots a_{r-1}, \ldots, a_{r-1}\right), \quad\left|a_{l}\right| \leq c_{l},
$$

for suitable $c_{i}$. Since

$$
\delta_{r_{m}}\left[\left(\begin{array}{ll}
a & 0 \\
0 & 1
\end{array}\right)\right]=\delta_{r_{m-1}}(a)|\operatorname{det} a|,
$$


we are reduced to considering the integral $\int_{c}|\operatorname{det} a|^{1-v} d^{\times} a$. This is indeed finite, provided $0<v<1$. Our argument shows in fact that, if in

$$
\int_{N_{r_{m-1}} \backslash G_{r_{m}-1}} F W^{\prime}\left[\left(\begin{array}{cc}
h & 0 \\
0 & 1
\end{array}\right)\right] d h
$$

we replace $F$ by its expression (11), then the resulting double integral converges. Thus (17) can be written as

$$
\begin{array}{r}
\int \psi(\operatorname{tr}(\varepsilon g x)) d x \int f^{*}\left[w^{*}\left(\begin{array}{ll}
1_{r_{m}} & x \\
0 & 1_{r-r_{m}}
\end{array}\right) p ; e,\left(\begin{array}{ll}
h & 0 \\
0 & 1
\end{array}\right) g\right] \\
\cdot \overline{W^{\prime}}\left[\left(\begin{array}{ll}
h & 0 \\
0 & 1
\end{array}\right)\right] d h .
\end{array}
$$

Next, since we have taken $u_{m}=0$, the representation $\pi_{m}$ of $G_{r}$ is pre-unitary. Thus (2.1.2) defines an invariant Hermitian form on थ $\left(\pi_{m} ; \psi\right)$. Hence the inner integral in (18) can also be written as

$$
\int f^{*}\left[w^{*}\left(\begin{array}{ll}
1_{r_{m}} & x \\
0 & 1_{r-r_{m}}
\end{array}\right) p ; e,\left(\begin{array}{ll}
h & 0 \\
0 & 1
\end{array}\right)\right] \bar{W}^{\prime}\left[\left(\begin{array}{ll}
h & 0 \\
0 & 1
\end{array}\right) g^{-1}\right] d h .
$$

Since $W^{\prime}$ is arbitrary we can replace $W^{\prime}$ by any of its right translates. We get that

$$
\begin{aligned}
\int \bar{W}^{\prime}\left[\left(\begin{array}{cc}
h & 0 \\
0 & 1
\end{array}\right)\right] d h \int \psi(\operatorname{tr}(\varepsilon g x)) d x \\
\cdot \int f^{*}\left[w^{*}\left(\begin{array}{ll}
1_{r_{m}} & x \\
0 & 1_{r-r_{m}}
\end{array}\right) p ; e,\left(\begin{array}{ll}
h & 0 \\
0 & 1
\end{array}\right)\right]=0
\end{aligned}
$$

for all $g \in G_{r_{m}}$ and all $p \in P_{r}$. Here $W^{\prime}$ can be taken arbitrary in $\mathscr{K}_{0}(\psi)$ (cf. (2.2.1)). Thus we finally get

$$
\int \psi(\operatorname{tr}(\varepsilon g x)) d x f^{*}\left[w^{*}\left(\begin{array}{ll}
1_{r_{m}} & x \\
0 & 1_{r-r_{m}}
\end{array}\right) p ; e, e\right]=0
$$

again for all $g \in G_{r_{m}}$ and $p \in P_{r}$. But $\operatorname{tr}(\varepsilon g x)=y x_{1}$, where $y$ is the last row of $g \in G_{r_{m}}$ and $x_{1}$ is the first column of $x$. Thus we get at first for all $y \in F^{r_{m}}$ nonzero, and then for all $y$, the relation

$$
\int \psi\left(y x_{1}\right) f^{*}\left[w^{*}\left(\begin{array}{ll}
1_{r_{m}} & x \\
0 & 1_{r-r_{m}}
\end{array}\right) p ; e, e\right] d x=0
$$


Since the integral (21) is absolutely convergent, we may apply Fourier inversion to conclude that

$$
\int f^{*}\left[w^{*}\left(\begin{array}{lll}
1_{r_{m}} & 0 & x \\
0 & 1 & 0 \\
0 & 0 & 1_{r-r_{m}-1}
\end{array}\right) p ; e, e\right] d x=0
$$

for all $p \in P_{r}$.

We shall now prove that, for any $j$ with $1 \leq j \leq r-r_{m}-1$, the relation

$$
\int f^{*}\left[w^{*}\left(\begin{array}{lll}
1_{r_{m}} & 0 & x \\
0 & 1_{j} & 0 \\
0 & 0 & 1_{r-r_{m}-j}
\end{array}\right) p ; e, e\right] d x=0
$$

for all $p \in P_{r}$, implies the same relation with $j$ replaced by $j+1$. For this let

$$
p^{\prime}=\left(\begin{array}{ll}
g & 0 \\
0 & 1_{r-r_{m}-J}
\end{array}\right),
$$

where $g$ is an element of $G_{r_{m}+j}$ of the form

$$
g=\left(\begin{array}{lll}
1_{r_{m}} & 0 & 0 \\
0 & 1_{j-1} & 0 \\
z & 0 & 1
\end{array}\right),
$$

$z$ being a row of length $r_{m}$. Our hypothesis on $j$ implies $p^{\prime} \in P_{r_{m}}$. Thus we can replace $p$ by $p^{\prime} p$ in (23). Then, after a simple computation, we get

(24) $\int f^{*}\left[\left(\begin{array}{lll}1_{j} & v x & 0 \\ 0 & 1_{r-r_{m}-j} & 0 \\ 0 & 0 & 1_{r_{m}}\end{array}\right) w^{*}\left(\begin{array}{lll}1_{r_{m}} & 0 & x \\ 0 & 1_{j} & 0 \\ 0 & 0 & 1_{r-r_{m}-j}\end{array}\right) p ; e, e\right] d x=0$.

Here $v$ is the $r_{m} \times j$ matrix given by

$$
v=\left[\begin{array}{c}
0 \\
-z
\end{array}\right] \text {. }
$$

Since $f^{*}$ belongs to the space of $\xi^{*}$, this reduces to the relation

$$
\int f^{*}\left[w^{*}\left(\begin{array}{lll}
1_{r_{m}} & 0 & x \\
0 & 1_{j} & 0 \\
0 & 0 & 1_{r-r_{m}-j}
\end{array}\right) p ; e, e\right] \psi\left(-z x_{1}\right) d x=0
$$


as before $x_{1}$ is the first column of $x$. If we again use Fourier inversion, we arrive at (23) with $j$ replaced by $j+1$.

Thus we have now proved that $f^{*}\left[w^{*} p ; e, e\right]=0$ for all $p \in P_{r}$. Replacing $p$ by

$$
\left(\begin{array}{ll}
1_{r_{m}} & 0 \\
0 & g
\end{array}\right) p, \quad g \in P_{r-r_{m}},
$$

we get

$$
f^{*}\left[w^{*} p ; g, e\right]=0
$$

for all $g \in P_{r-r_{m}}, p \in P_{r_{m}}$. Since the function $g \mapsto f^{*}\left[w^{*} p ; g, e\right]$ belongs to W $\left(\xi^{\prime} ; \psi\right)$, at this point we can apply the second part of our induction hypothesis to the representation $\xi^{\prime}$ to conclude that

$$
f^{*}\left[w^{*} p ; g, e\right]=0
$$

for all $p \in P_{r_{m}}$ and now for all $g \in G_{r-r_{m}}$. But then (27) implies that $f^{*}\left[u w^{*} q ; e, e\right]=0$ for all $q$ in the parabolic subgroup of type $\left(r_{m}, r-r_{m}\right)$ and all $u$ in the unipotent radical of $Q^{*}$. By continuity we get $f^{*}[g ; e, e]=0$ for all $g$, that is, $f=0$.

\section{REFERENCES}

[B-Z] I. N. Bernstein and A. V. Zelevinsky, Induced representations of reductive p-adic groups, Ann. Scient. Éc. Norm. Sup., 4 e série, t. 10 (1977), 441-472.

[B-W] A. Borel and N. Wallach, Continuous cohomology, discrete subgroups, and representations of reductive groups, Annals of Math. Studies, No. 94, Princeton University Press, 1980.

[G-J] S. Gelbart and H. Jacquet, A relation between automorphic representations of GL(2) and GL(3), Ann. Scient. Éc. Norm. Sup., $4^{\mathrm{e}}$ série, t. 11 (1978), 471-542.

$[\mathrm{G}-\mathrm{K}] \quad$ J. M. Galfand and D. A. Kazdan, Representations of $\mathrm{GL}(n, K)$ where $K$ is a Local Field, in Lie Groups and Their Representations, John Wiley and Sons, 1975, pp. 95-118.

[J] H. Jacquet, Generic Representations, in Non-commutative harmonic analysis, Marseille-Luminy, 1976, Springer-Verlag, Lecture Notes No. 587, (1976), 376378.

[J-P-S] H. Jacquet, I. I. Piatetski-Shapiro, and J. Shalika, Automorphic forms in GL(3) I, II, Annals of Math., 109 (1979), 169-212 and 213-518.

[J-P-S] __ On Rankin Selberg convolution, to appear.

[K] B. Kostant, On Whittaker vectors and representation theory, Inventiones Math., 48 (1978), 101-184. 
[V] D. A. Vogan, Gelfand-Kirillow dimension for Harish-Chandra modules, Inventiones Math., 48 (1978), 75-98.

[Z] A. V. Zelevinsky, Induced representations of reductive p-adic groups II, Ann. Scient. Éc. Norm. Sup., $4^{\text {e }}$ série, t. 13 (1980), 165-210.

Received October 10, 1981 and in revised form July 19, 1982. This research was partially supported by National Science Foundation Grants: MCS 79-01712 and MCS 79-13799.

Columbia University

New York, NY 10027 


\section{PACIFIC JOURNAL OF MATHEMATICS}

EDITORS

Donald BABBITt (Managing Editor)

University of California

Los Angeles, CA 90024

Hugo Rossi

University of Utah

Salt Lake City, UT 84112

C. C. Moore and Arthur Ogus

University of California

Berkeley, CA 94720

\section{J. DugundiI}

Department of Mathematics

University of Southern California

Los Angeles, CA 90089-1113

R. Finn and H. SAMELSON

Stanford University

Stanford, CA 94305

\section{ASSOCIATE EDITORS}
R. ARENS
E. F. BECKENBACH
B. H. NEUMANN
F. WOLF
K. YOSHIDA (1906-1982)

\section{SUPPORTING INSTITUTIONS}

UNIVERSITY OF ARIZONA

UNIVERSITY OF BRITISH COLUMBIA

CALIFORNIA INSTITUTE OF TECHNOLOGY

UNIVERSITY OF CALIFORNIA

MONTANA STATE UNIVERSITY

UNIVERSITY OF NEVADA. RENO

NEW MEXICO STATE UNIVERSITY

OREGON STATE UNIVERSITY
UNIVERSITY OF OREGION

UNIVERSITY OF SOUTHERN CALIFORNIA

STANFORD UNIVERSITY

UNIVERSITY OF HAWAII

UNIVERSITY OF TOKYO

UNIVERSITY OF UTAH

WASHINGTON STATE UNIVERSITY

UNIVERSITY OF WASHINGTON 


\section{Pacific Journal of Mathematics}

Vol. 109, No. $1 \quad$ May, 1983

Donald George Babbitt and V. S. Varadarajan, Formal reduction theory of meromorphic differential equations: a group theoretic view $\ldots \ldots \ldots \ldots 1$

Jo-Ann Deborah Cohen, Norms on $F(X) \ldots \ldots \ldots \ldots \ldots \ldots \ldots \ldots \ldots \ldots$

Robert Fitzgerald, Witt kernels of function field extensions $\ldots \ldots \ldots \ldots \ldots 89$

Hervé Jacquet and Joseph Andrew Shalika, The Whittaker models of induced representations .............................. 107

Masakiti Kinukawa, Some generalizations of contraction theorems for

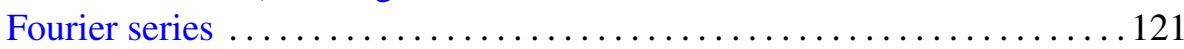

Joseph Weston Kitchen, Jr. and David A. Robbins, Sectional

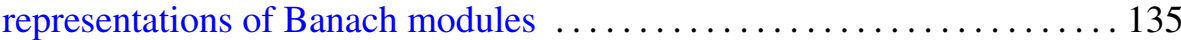

Victor Charles Pestien, Jr., Weak approximation of strategies in measurable gambling

Richard Scott Pierce and Charles Irvin Vinsonhaler, Realizing central

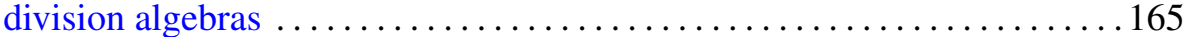

Walter Ricardo Ferrer Santos, Cohomology of comodules ............ 179

Marko Tadić, Harmonic analysis of spherical functions on reductive groups

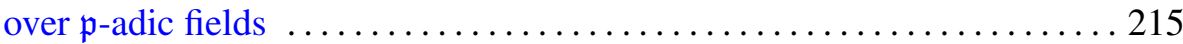

Lorenzo Traldi, The determinantal ideals of link modules. II . . . . . . . . 237

Alain J. Valette, A remark on the Kasparov groups $\operatorname{Ext}^{i}(A, B) \ldots \ldots \ldots 247$ 